the filtrate treated with $I_{\frac{1}{2}}$ gramme of chloride of ammonium, and boiled and allowed to stand, whereupon the alumina is precipitated as phosphate of alumina, which admits of being got on a filter, washed, ignited, and weighed.

The advantage gained in this instance by using weighed quantities of reagents, and by avoiding dilution, will commend itself to chemists, who will not fail to recognise that, though there be difficulty in insuring that an indefinite quantity of acid and alkali shall be quite free from alumina and silica, there is not much difficulty in getting $1 \mathrm{gramme}$ of sulphuric acid, $1 \frac{1}{2}$ gramme of potash or soda, and $1 \frac{1}{2}$ gramme of chloride of ammonium, so as collectively not to contain a couple of millisrammes of alumina or of silica.

The testing whether flour be sound or not by the strength of the aqueous extract is not new, but has been developed and rendered easily practicable in the course of my experiments. The basis of the method is, that in sound flour there is very little sugar and dextrine, but that in unsound flour there is either much sugar and dextrine, or else that a short exposure to the action of water converts much of the starch of the flour into dextrine and sugar. In the space of an hour and a half, I can by a little management make a determination of the quantity of extractive given by a sample of flour. I take roo grammes of the flour, mix it well with some water, and then dilute the whole mass with water until it occupies exactly half a litre. I then pour it on a dry paper filter in a dry funnel, whereupon the liquid runs through tolerably rapidly at first ; by and by, as is known, such a filter will become clogged up, but not until at least some 50 cubic contimètres of filtrate have run through. These may be measured and evaporated down to dryness in the waterbath, and the residue weighed. Ten times the residue yielded by the 50 cubic centimetres is equal to the amount of extractive yielded by 100 grammes of the flour. The extractive furnished by 100 grammes of sound flour is 4.69 grammes. Of this, $0.44 \mathrm{gramme}$ is ash and 0.92 gramme vegetable albumen, leaving 3.33 grammes of dextrine, sugar, and gum. If sound flour be left for twenty hours in contact with cold water, and then the aqueous extract be taken, it will be found to be increased somewhat : I found it to be 6.01 srammes. In unsound flour, Odling has found 12 and 18.2 grammes of iextractive per 100 grammes of flour. I believe that Odling first proposed the determination of the amount of extractive yielded by flour to cold water as a test of the soundness of flour. I am inclined to the belief that this test may be made very practicable and valuable.

\section{METHYLENE ETHER AS AN ANÆSTHETIC.} \section{By J. O. BROOKHOUSE, M.D., Surgeon to the Eye Infirmary,
Nottingham.}

SINCE a more prominent professional attention has been generally directed to the subject of anæsthetics, in consequence of the number of recent fatal accidents with chloroform, I have made use of Dr. Richardson's new compound, methylene ether $\left(\mathrm{C}^{2} \mathrm{H}^{5}\right)^{2}, \mathrm{C} \mathrm{H}^{2} \mathrm{Cl}^{2}$, specific gravity 1000, prepared by Messrs. Hearon, Squire, and Francis, of Coleman Street, London. The opinion formed is deduced from a limited number of cases, and wo uld have been valueless, by reason of such limitation, had it not happened that the special lethal influence of the agent was manifested on more than one occasion.

The ether was given on a wire skeleton mask covered with one thickness of flannel closely fitting the nose and mouth; over this was then laid one fold of an ordinary towel. The majority of the patients were under twenty years, and all free from vital organic disease. The following are some of the cases and facts observed.

CASE I. - H. B., aged 26, married woman. About three drachms of ether were used. Anæsthesia was produced in three minutes. The time of operation was two minutes (the operation was for closed pupils). The pulse was I04 before, during and after operation. Respiration was unaffected. The facial aspect was unchanged. There was no excitement. The time of recovery was four minutes. The sickness was very slight.

CASE II.-C. Y., aged I7, single woman. About three drachms of ether were used. Anæesthesia was produced in two minutes. The time of operation was six minutes. The pupils were medium-sized and fixed. The pulse before operation was 84 ; it fell to 60 during the anæsthesia, and became intermittent. The respiration was slow and very feeble. The face was pale. Excitement was small, She recovered in four minutes, and was subsequently very sick. The symptoms shown by this girl were most alarming.

CASE III.-W. S., a boy aged 13. About three drachms of ether were used. Anæsthesia was produced in three minutes; he was under its influence three or four minutes, when the pulse fell away quicklyI 4 beats per minute. He was allowed to recover, when the pulse rose 20. He was put under a second time, without any alteration of pulse, rate or quality. His face was flushed; the lips purple in colour. The excitement was very slight. The time of recovery was four minutes. He was afterwards sick.

CASE IV.-L. T., a girl aged II. About one drachm of ether was used. Anæsthesia was produced in two minutes. The pulse was 100 before and during insensibility. The excitement was very slight. Recovery was quick. The face was flushed; the pupils contracted. This girl had the ether three days before, and took it well.

CASE V.-R. C., a boy aged 5. About two drachms of ether were used. Anæsthesia was produced in a minute and a half. The time of operation was three minutes. There was no excitement. The pupils were medium-sized and fixed. The pulse was i 20 before employing ether (this boy was nervous and frightened); it fell 16 per minute during insensibility. The respiration was unaffected. The face was pallid. He recovered in five minutes.

CASE vi.-C. M., a girl aged 15. About four drachms of ether were used. The time in producing anæsthesia was eight or ten minutes; of operation, five minutes. The pulse before the operation was 104; during the inhalation of ether, it fell to 78 , and was unsatisfactory in quality. The respiration was not specially affected. The face was suffused and purple, with marked capillary blood-stasis. Recovery was quick. He was subsequently sick. This patient did not take the ether well. As soon as she began to get fully under its influence, her pulse fell away; whilst, if only under it in a degree hardly less than dangerous, she struggled. She was a fine, well developed girl, and, during partial unconsciousness, manifested feelings of a sensuous character.

CASE VII. - C. B.. a girl aged 16. About three drachms of ether were used. Anæesthesia was produced in five minutes; she was under its influence eight minutes. The excitement was small. The pupils were medium-sized and fixed. The pulse before the operation was 96 ; it fell to 68, and was small and weak. The respiration was not specially affected. The face was suffused and purple; capillary congestion was marked. She recovered quickly, and was subsequently sick.

All the above patients were in the recumbent posture, and their dress was loosened about the neck and chest.

It appears clearly, therefore, from these cases, that this ether is a direct paralyser of the heart, and not certainly in any less degree than chloroform. This, indeed, so far as I can judge, is its only objection; whilst in many ways it possesses advantages. It is quicker in its action and time of recovery. Sickness, although almost always. present, is not so persistent ; and the patient does not complain of feeling so altogether "seedy". It does not blister the skin. The leading question, however, is the question of comparative safety; and if this, as I fear, cannot be answered satisfactorily, the anxious expectancy of the profession and public is still indefinitely continued.

\section{THERAPEUTIC MEMORANDA.}

\section{CARBOLIC ACID DRESSINGS.}

I HAVE watched with great interest the recent communications in your paper on the above subject, and am bound to say I feel considerable difficulty in explaining to myself the different statements regarding this liberty to give my experiences on the matter? For the last five or six years I have used carbolic acid in almost every possible form and under the most varying circumstances, and always with the most happy results. In wounds, accidents, and surgieal operations, I invariably use it either in the form of lotion or mixed with oil, or the acid undiluted. In the latter case, I consider it acts as a caustic; and in all cases where $I$ have had a dirty unhealthy wound to deal with $I$ have found it to serve my purpose well. Recently I used the pure acid in a case where sloughing took place after the operation (flap) on the leg. Under its use in a short time the sloughs were thrown off, and the wound filled up rapidly with healthy granulations. The stump is now progressing most favourably under a dressing of carbolic acid and oil (one pint in twelve). In this case there is no perceptible change in the urine. In conclusion, I beg to state that I have never observed the slightest symptom of injurious action in all my varied experience in the use of this valuable agent. E. CROCKETT,
yfarthfa Ironworks. 